\title{
On the Use of a Powder Rheometer to Characterize the Powder Flowability at Low Consolidation with Torque Resistances
}

\author{
Hamid Salehi, Diego Barletta, and Massimo Poletto \\ Dipartimento di Ingegneria Industriale, Università degli Studi di Salerno, Via Giovanni Paolo II, 132, I-84084 Fis- \\ ciano (SA), Italy \\ Denis Schütz and Richard Romirer \\ Development Rheometry, Anton Paar GmbH, Anton Paar Strasse 20, Graz, Austria
}

\begin{abstract}
The Anton Paar Powder Cell was used to measure the torque necessary to rotate an impeller in beds of glass beads, sand and alumina powders aerated between no aeration to the minimum for fluidization. Measured torque values depend on the material tested, on the air flow rate applied, on the impeller depth and on the height of the impeller blade. The effect of the impeller depth is linear for low impeller depth and is less than linear at high depth values. A model was developed for the interpretation of the experimental results based on the idea that the material is shearing on the surface described by the impeller rotation. The model allows to estimate an effectiveness of the impeller in the torque determination and also to predict the torque for the impeller at the at deepest positions at which the wall effects have to be considered.
\end{abstract}

Keywords: aerated powders, torque measurement and estimation, powder flowability, powder cell, low consolidation

\section{Introduction}

In some industrial applications bulk solids must flow under stresses smaller than few Pascals, such as in small process hoppers, mixers of pharmaceutical powders, ${ }^{1,2}$ toner cartridges,${ }^{3}$ dosing and dispersion in dry powder inhalers, ${ }^{4}$ dosing of food powders. ${ }^{5}$ Hence, it can be useful to know the powders flow properties in the low stress ranges. ${ }^{6}$ Conventional shear testers are not designed to allow measurements in low stress ranges. ${ }^{7,8}$ For instance, stresses around $100 \mathrm{~Pa}$ require an exact evaluation of the contribution of powder layers of a few millimeters. It should be considered that, additional stresses in conventional apparatus may arise as a consequence of the weight of shear testers parts that press the bulk solid specimen, ${ }^{9}$ unless special procedures are adopted. ${ }^{10}$ A lower limit for a typical Jenike-type shear tester is a consolidation stress of about $3 \mathrm{kPa}$, whereby the ring shear tester allows testing at smaller stresses. ${ }^{9}$ In particular, Schulze and Wittmaier ${ }^{11}$ attained consolidation stresses lower than $500 \mathrm{~Pa}$ in annular shear cells by applying small normal loads and increasing the sensitivity of load cells. In other researches, low consolidation stress ranges are attained by introducing a gas pressure gradient opposed to gravity through the powder sample. ${ }^{12-14}$
It has been observed that the reproducibility of flowability tests results is greatly reduced at low levels of the major principal stress (generally less than a few $\mathrm{kPa}$ ), and/or the measured values seem inconsistent with observed flow behaviors at the conventional shear testers. ${ }^{15}$ In addition, materials which these testers find to be cohesion-less ( $\mathrm{fc}=\sim 0$ ) may have practical differences in flow behavior, especially in regions of low consolidation stress. ${ }^{16}$

The alternative techniques used to study the rheological properties of powders under loose conditions are the derivation of the tensile strength and yield loci from fluidization experiments $^{17,18}$ and the derivation of cohesion and angle of friction from observations of the avalanching behavior of powders. ${ }^{19}$ Another method, the ball indentation method on a bed of cohesive powders, developed by Zafar et al., ${ }^{20}$ to assess the flowability of a small quantity of powders at very low stress levels. This method is not able to measure the tensile strength directly, but it provides a method to assess the resistance of the powder bed to plastic deformation. Zafar et al., ${ }^{20}$ also proposed as an alternative the use of the raining bed method, in which powder compaction is obtained by means of gas pressure drops in a fixed bed. Studies on the rheological behavior of powder flows at low consolidations were performed also by rotational rheometers of different geometries, like the FT4 Powder Rheometer. ${ }^{21}$ New methods such as the use of fluidization were qualitatively compared with traditional methods suggesting a promising perspective for powder rheometry. ${ }^{22,23}$ Recently, the Discrete Element Method (DEM) was also applied to model the dynamic behaviour of the FT4 Powder 
Rheometer to predict the flow energy measured by the impeller under nonaerated conditions ${ }^{24}$ and in presence of an air flow through the powder bed by coupling DEM with Computational Fluid Dynamics for the gas flow. ${ }^{25}$

A mechanically stirred fluid-bed rheometer (msFBR) was developed by Bruni et al. ${ }^{21}$ at the University College of London (UCL) to study the rheology of aerated and fluidized powders. Consequently, Bruni et al. ${ }^{26}$ developed a model based on the method of differential slices (Janssen's approach ${ }^{27}$ ) and on a Mohr-Coulomb description of the powder rheology to describe the powder stress state and the applied torque in the msFBR. This instrument proved to be able to measure powder flow properties at low levels of consolidation stress, thanks to the combined actions of material weight and vertical aeration, as well as to the different depth at which the impeller can be placed.

A similar instrument was developed by Anton Paar Company to measure powder flowability in partially or totally suspended conditions in presence of vertical air flow. The Anton Paar Powder Cell (APPC) instrument derived from Anton Paar viscometers, in which it is possible to precisely measure the torque necessary to rotate an impeller immersed in a vertically aerated powder either below or above the minimum for fluidization. This instrument is interesting for the high torque measurement precision on extremely wide measuring ranges spanning on several decades. In fact, this feature, together with the possibility to aerate the powder sample, makes the instrument suitable to measure flow properties at very low consolidation values. In spite of the several similarities between the UCL msFBR and the Anton Paar Powder Cell, the two apparatuses differ significantly for the size ratio between the impeller and the diameter of the fluidized bed, 36/ $140 \approx 0.26$ for the msFBR and $30 / 50=0.6$ for the powder Cell. This difference may introduce significant differences also in the relevant phenomenology of the two testers and, therefore, the interpretation framework found for the msFBR may not be adequate for the APPC. This article, therefore, aims at verifying the possibility to relate the torque to the powder flow properties commonly used to characterize the powder flowability. In particular, rather than identifying alternative procedures to use the measured torques to estimate some of the powder flow properties, this paper will try to identify the correct framework of physical interpretation of the phenomena observed. Such step is necessary to extend the use of rheometers beyond the well-established applications of product quality control and to suggest the proper system configuration and measurement procedure. Three materials of different particle size were used in the experiments. The materials showed a slight different flowability, but they are all in the range of free flowing powders. The objective was to explore flow conditions not affected by powder cohesion and, in particular, in which the effective angle of powder internal friction did not change significantly with consolidation. The torque measurements were performed in experiments under stationary impeller rotation at a fixed low speed. The main operating variables explored were the air flow rate and the impeller positon.

\section{Experimental}

\section{Apparatus}

The Anton Paar Powder Cell (Figure 1) is a set up by which it is possible to couple the Anton Paar rheometer bench with a powder sample, hosted in a $50 \mathrm{~mm}$ ID glass cylinder in which
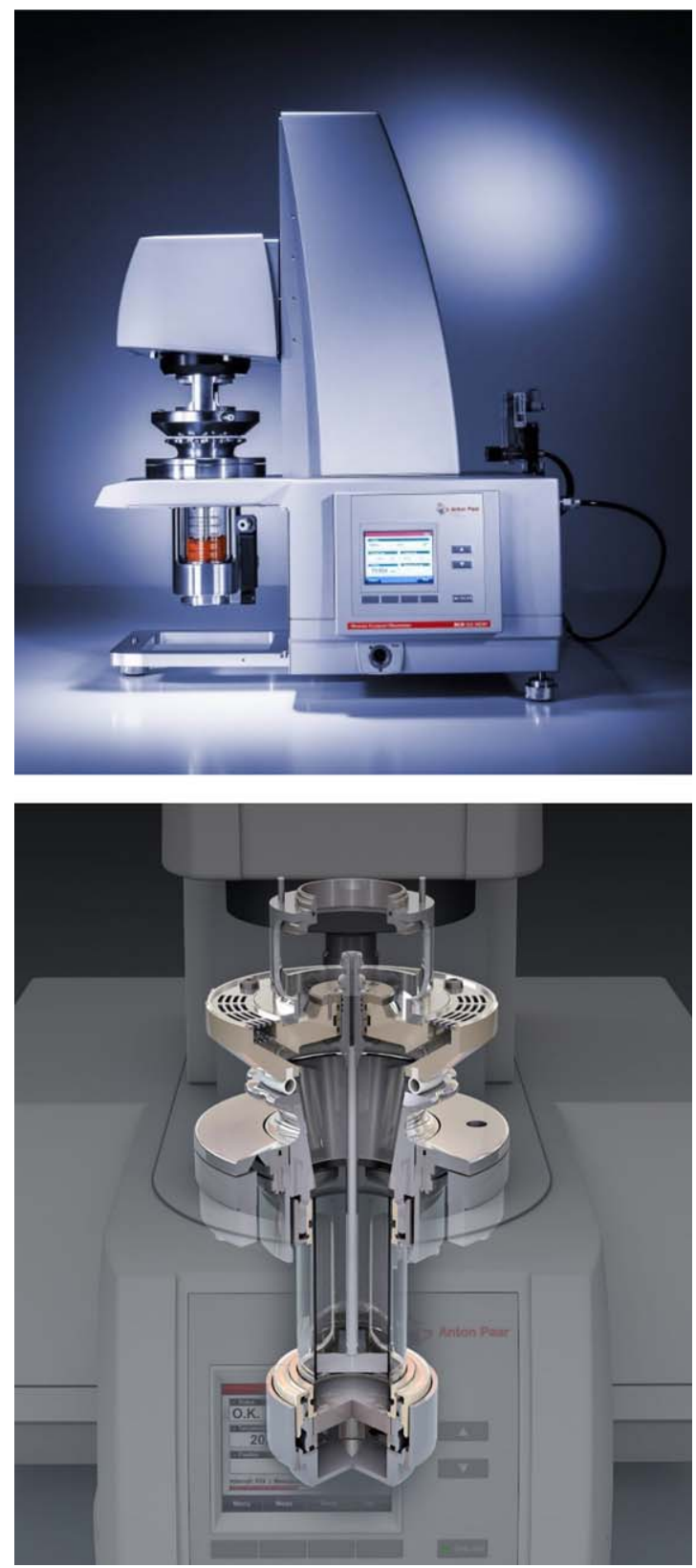

Figure 1. The Anton Paar Powder Cell (Copyright by Anton Paar).

(a) the MCR rheometer with the Powder Cell; (b) a sketch of the Powder Cell. [Color figure can be viewed at wileyonlinelibrary.com]

the powder can be aerated below and above the minimum for fluidization. The rheometer can be equipped with impellers of different geometries, such as cylinders or double blade turbines. The torque sensor installed on the Anton Paar rheometer is able to measure torques in a range of several decades between $10^{-8} \mathrm{Nm}$ and $0.3 \mathrm{Nm}$, and therefore allows to continuously measuring torques in powders from below to above the minimum air velocity for fluidization. The impeller rotational speed can be regulated between $0.1 \mathrm{rpm}$ and $500 \mathrm{rpm}$. The gas distributor at the cell bottom is a frit made of a 5-mm thick circular porous plate of fine sintered ceramic materials, characterized by very high uniform flow resistance that, therefore, is able to ensure a uniform gas distribution even using fine powders. The gas flow rate can be regulated by an external mass 
Table 1. Materials Properties, Sample Masses, and other Model Parameters

\begin{tabular}{|c|c|c|c|c|c|c|c|c|c|c|c|}
\hline Material & $\begin{array}{c}d_{\mathrm{p} 3,2} \\
\mu \mathrm{m}\end{array}$ & $\begin{array}{l}d_{\mathrm{p} 50} \\
\mu \mathrm{m}\end{array}$ & $\begin{array}{c}d_{\mathrm{p} 10 \_} d_{\mathrm{p} 90} \\
\mu \mathrm{m}\end{array}$ & $\begin{array}{c}\rho_{\mathrm{b}} \\
\mathrm{kg} \mathrm{m}^{-3}\end{array}$ & $\begin{array}{c}U_{\mathrm{mf}} \\
\mathrm{cm} \mathrm{s}^{-1}\end{array}$ & $\begin{array}{l}\mathrm{M} \\
\mathrm{kg}\end{array}$ & $\begin{array}{c}\delta \\
\operatorname{deg}\end{array}$ & $\begin{array}{l}\phi_{\text {we }} \\
\text { deg }\end{array}$ & $\begin{array}{c}\phi_{\mathrm{wm}} \\
\mathrm{deg}\end{array}$ & $\begin{array}{c}H_{\mathrm{r}} \\
\mathrm{m}\end{array}$ & $\eta$ \\
\hline Glass beads & 151 & 163 & $120-223$ & 2500 & 0.66 & 0.225 & 22 & 14.3 & 15.8 & 0.030 & 0.42 \\
\hline Sand & 225 & 266 & $190-370$ & 2500 & 1.38 & 0.23 & 30.5 & 17.5 & 33.8 & 0.034 & 1.00 \\
\hline Alumina & 75 & 80 & 53-119 & 1730 & 0.14 & 0.16 & 31.5 & 19.4 & 29.3 & 0.033 & 0.60 \\
\hline
\end{tabular}

flow meter, chosen as the most appropriate to operate in the flow range for the tested powder. An absolute pressure transducer is used to measure absolute gas pressures upstream the frit.

The blade operating position is placed at a height which ensures a $10-\mathrm{mm}$ gap from the cell base with the standard impeller, bearing a flat blade $30-\mathrm{mm}$ long from tip to tip and 10 -mm high. The impeller can be moved vertically, to assume any position along the axis of the cylindrical container above the $10-\mathrm{mm}$ minimum gap position. The Apparatus can be programmed by a custom built in software interface with defined sequences of operation.

The experiments reported in this article were made with both a standard impeller and a customized impeller in which the blade differed from the standard only for the blade height that was $5 \mathrm{~mm}$. Two air flow meters were used a medium capacity mass flow meter, $15.0 \mathrm{~L} / \mathrm{min}\left(2.510^{-4} \mathrm{~m}^{3} \mathrm{~s}^{-1}\right)$, in standard conditions and a low capacity mass flow meter, $5.00 \mathrm{~L} / \mathrm{min}\left(8.3310^{-5} \mathrm{~m}^{3} \mathrm{~s}^{-1}\right)$ in standard conditions.

\section{Materials}

Three free flowing powders, according to Jenike classification, ${ }^{9}$ namely, Glass beads, Sand, and Alumina powders were used. The most relevant properties of these powders were measured using a Malvern Mastersizer 2000, with concern to the particle-size distribution, and using a Brookfield Powder Flow Tester (PFT), with concern to the flow properties. The results are reported in Table 1 . It has to be noted that it was not possible to have available a flat sample of the powder cell to measure the wall friction and, therefore, measurements were performed on a glass wall sample cut from a commercial glass plate.

Glass beads are a model material characterized by particles with high sphericity and relatively narrow particle-size distribution. Sieved silica sand is a "real" particulate material characterized by properties very similar to glass beads, but with particles of a slightly more irregular shape and surface than glass beads, a much larger particle-size distribution and angle of internal friction. Alumina is a much finer and lighter material than the other two, but still, it is characterized by particles of high sphericity.

Desiccated air was used in Anton Paar Powder Cell to keep the powders moisture constant during tests.

\section{Methods}

The absolute pressure measured with the absolute pressure transducer in the wind-box upstream the frit. This requires the setup of a proper measurement procedure to estimate the relative gas pressure at the bottom of the glass cylinder, just above the frit, and equal to the gas pressure drops in the powder bed. In particular, the pressure calibration procedure consists in measuring the gas pressure in the wind-box in the empty cell as a function of the air rate. This function allows to subtract from the total pressure taken during the experiments and the sum of the pressure drop due to the frit and of the atmospheric pressure. The resulting calibrated pressure represents the true pressure drops in the bed.

Fluidization experiments were performed in the Anton Paar Powder Cell, to find out the minimum fluidization velocity $\left(U_{\mathrm{mf}}\right)$. A dedicated procedure was developed to carry out a fluidization and defluidization experiment in series. To obtain a reproducible fluidization curve a bed preconditioning sequence of operations was included in the procedure. In this sequence, the bed is brought to full fluidization and then it is let to settle by reducing the aeration rate at a controlled speed. Given the different ranges of gas rates for fluidization, the applied flow rates in the bed conditioning sequence depend on the material used. Fluidization conditions started with increasing gas rate ramps (Figure 2, curve branch a) of $20 \mathrm{~s}$ in which the gas flow rate was steadily increased from 0 and ending up to $5 \mathrm{~L} / \mathrm{min}\left(8.310^{-5} \mathrm{~m}^{3} \mathrm{~s}^{-1}\right), 10 \mathrm{~L} / \mathrm{min}\left(1.010^{-4} \mathrm{~m}^{3} \mathrm{~s}^{-1}\right)$, and $1.2 \mathrm{~L} / \mathrm{min}\left(2.010^{-5} \mathrm{~m}^{3} \mathrm{~s}^{-1}\right)$ for Glass beads, Sand, and Alumina, respectively. Then, the gas flow rate was left unchanged for $40 \mathrm{~s}$ (Figure 2, curve branch b) and finally it was brought to 0 in a $20 \mathrm{~s}$ decreasing gas rate ramp (Figure 2, curve branch c). At the end of the bed preconditioning sequence, the volumetric flow rate was increased to the first desired gas rate, set for the first point of fluidization curve, using a ramp of $20 \mathrm{~s}$ (Figure 2, curve branch d). Then the flow rate was held constant for $40 \mathrm{~s}$ (Figure 2, curve branch e), during this time the whole pressure signal was considered to evaluate the average pressure value to be used to place the first point of the powder fluidization curve. Then the flow was increased to the other set values of the gas rates to be used to obtain the fluidization curves using similar sequences of $20 \mathrm{~s}$ increasing gas rate ramps and $40 \mathrm{~s}$ holding periods. When, the

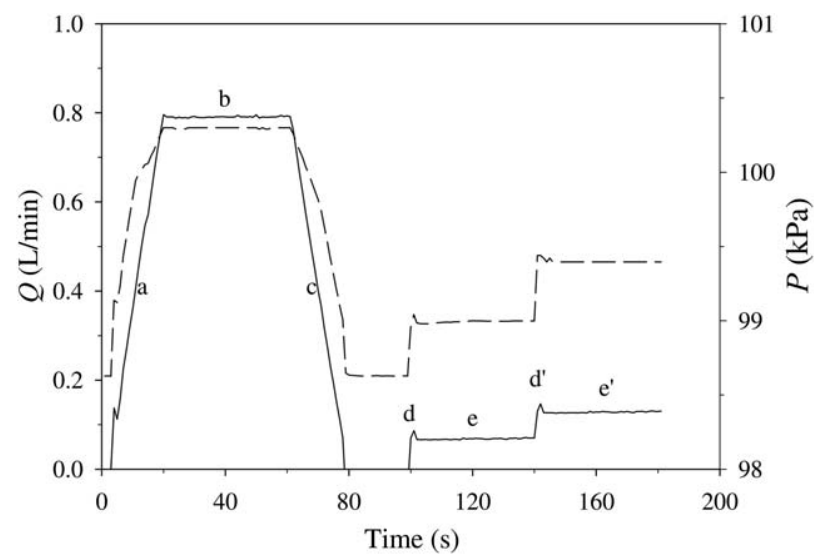

Figure 2. Example (Alumina case) of gas rate, $\mathbf{Q}(-)$, time profile for the measurement of a fluidization curve and the corresponding calibrated relative pressures at the bed bottom $\boldsymbol{P}(--)$.

Bed conditioning sequence: (a) ramp increase; (b) steady flow; (c) ramp decrease. Measurement sequence for a single point of the fluidization curve: (d) ramp increase; (e) steady flow for pressure measurement. 

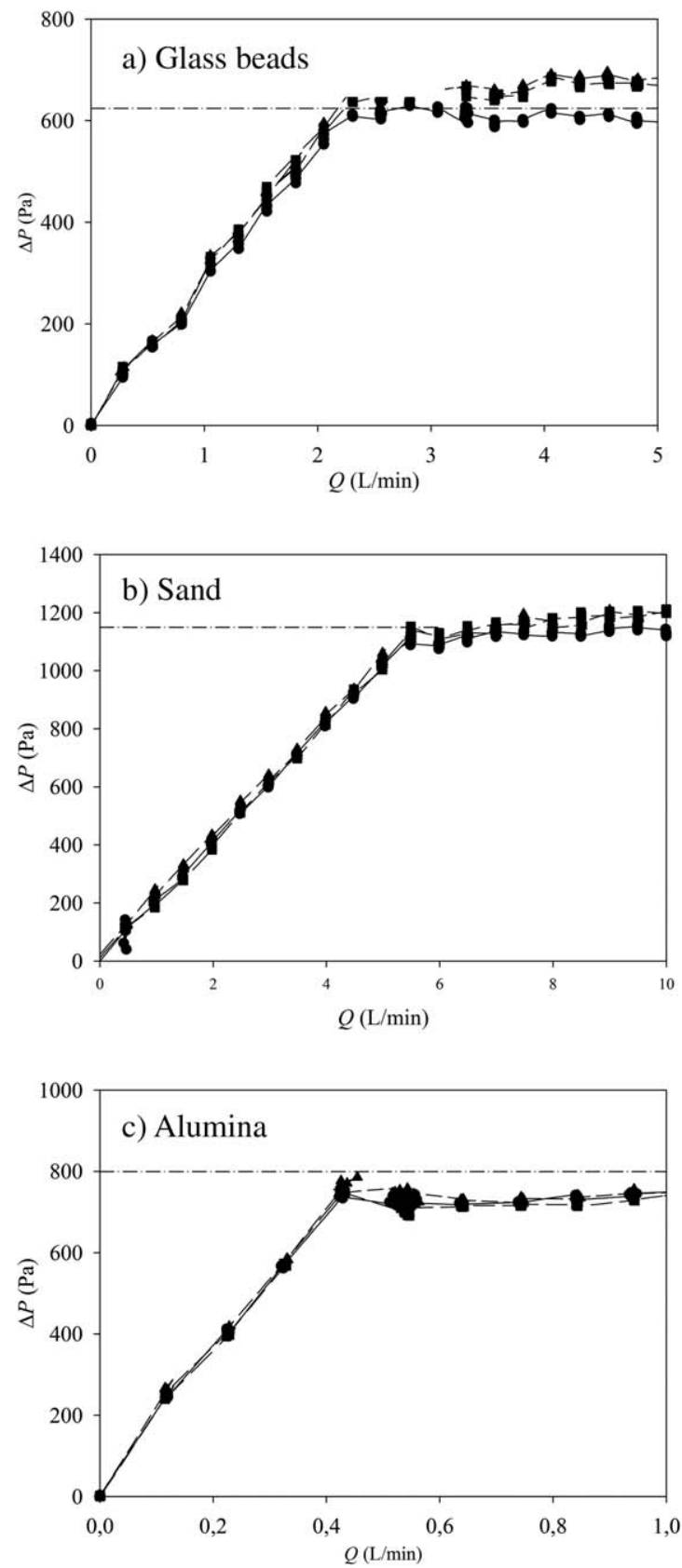

Figure 3. Fluidization curves for the different materials: $0,-$, Test $1 ; \mathbf{\square},--$, Test 2; $\Delta,---$, Test 3 ; - - , theoretical pressure drops at fluidization.

maximum value of the fluidization rate was obtained the same sequence of air flow rates was tested in the inverse order using sequences of $20 \mathrm{~s}$ decreasing gas rates ramps and $40 \mathrm{~s}$ holding periods. The change of gas flow rates with ramps was adopted to minimize the effect of changes of gas flow rate. It has to be acknowledge, however, that in the few test in which the ramp time was changed, or even eliminated at all, no significant change could be observed between values of the measured pressure drops.

A second dedicated automated procedure was developed to measure the torque necessary to rotate the impeller at a fixed rotational speed, as reported in Figure 4. Also in this case, a powder conditioning step was performed similarly to the measurement procedure above described for the fluidization curve. Then the flow rate was set to the desired value and the torque measured by rotating impeller at the fixed velocity of $0.5 \mathrm{RPM}$. A period of $170 \mathrm{~s}$ was allowed to reach to the steady state and the measure was taken in a following $240 \mathrm{~s}$ interval. The procedure then automatically moved the impeller to the subsequent measurement position, typically $5 \mathrm{~mm}$ above, where both the bed preconditioning step and the torque measurement step are wholly repeated.

\section{Results and Discussion}

Figure 2 reports the typical gas flow rate and the corresponding measured gas pressure time series for an alumina powder. It is clearly shown that the gas pressure transients are negligible in the constant gas velocity branches of the curve and, therefore, the whole set of data in this branch can be used to calculate the corresponding average gas pressure values at the set gas flow rates.

Figure 3 shows the results of three independent experiments for each of the materials tested compared with the theoretical values of the pressure drops for fluidization of the material tested. Repeatability of the results is fair for all the tests. The flow rates for minimum fluidization, found as the experimental intersection of the fixed bed branch and the fluidized bed branch of the fluidization curve, are $2.4 \mathrm{~L} / \mathrm{min}\left(4.010^{-5} \mathrm{~m}^{3}\right.$ $\left.\mathrm{s}^{-1}\right), 5.5 \mathrm{~L} / \mathrm{min}\left(8.310^{-5} \mathrm{~m}^{3} \mathrm{~s}^{-1}\right)$, and $0.5 \mathrm{~L} / \mathrm{min}\left(8.310^{-6}\right.$ $\mathrm{m}^{3} \mathrm{~s}^{-1}$ ) for Glass beads, Sand, and Alumina powders, respectively. The corresponding minimum fluidization velocities of the three powders are reported in Table 1.

The raw data of a typical torque experiment at a certain depth of the impeller are reported in Figure 4. From the figure, it is possible to verify that after a fixed flow rate is set the measured torque reaches the steady state after some time. Two kinds of time effect can be visualized, short time fluctuations having a period of about $10-20 \mathrm{~s}$ which are probably due to the onset of the shear envelope around the rotating impeller and longer time changes to be attributed to the more gradual bed compaction. The bed compaction takes place as a consequence of the stirring action on the bed produced with a defluidization procedure and, therefore starting from a rather loose state. A confirmation of this interpretation can be found from the results obtained with experiments in which the effect of the different impeller rotational speeds on the measured torque was assessed. Figure 5 reports the torque time series for Sand in the first $500 \mathrm{~s}$ after the start of the impeller rotation. It

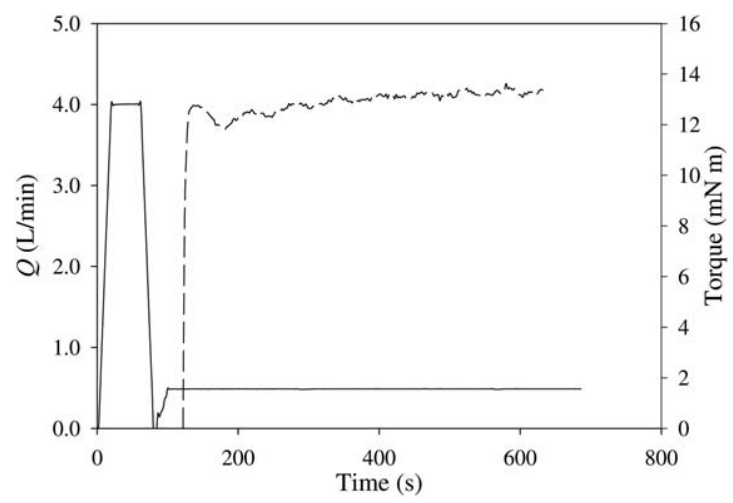

Figure 4. Example of time series if the gas rate profile and of the measured torque in a torque measurement test. $Q(-)$; Torque $(--)$ for Glass Beads powder. 


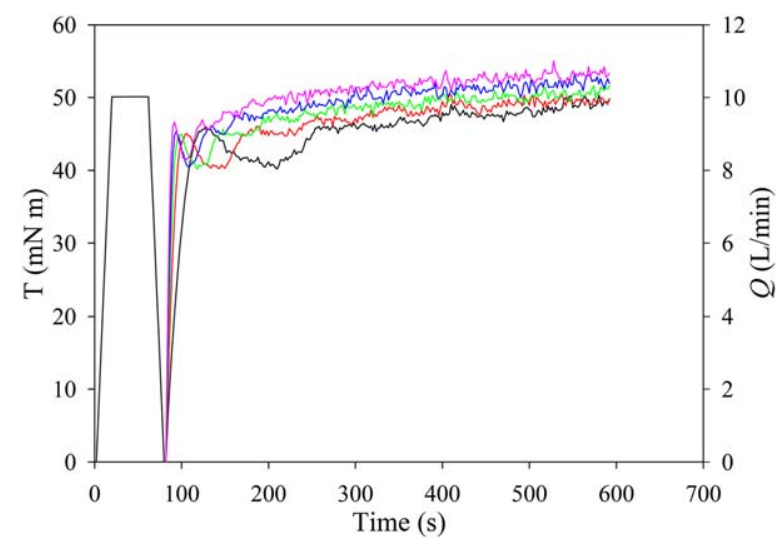

Figure 5. Example of torque time series showing the effect of impeller rotation speed on the measured torque in Sand after fluidization for different impeller rotation speeds: $0.2 \mathrm{rpm}$

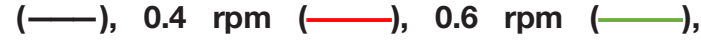
$0.8 \mathrm{rpm}(\longrightarrow), 1 \mathrm{rpm}(-)$.

[Color figure can be viewed at wileyonlinelibrary.com]

is clearly visible from the figure that the higher the speed the faster are the torque changes as it should be expected by the higher dissipated power for higher rotational speeds. It is also possible to see from the figure that increasing the impeller rotation speed makes the torque signal more stable. As we aim at comparing the results of this study with those obtained in shear testers working at very low shear rates, a value of 0.5 RPM was used in all the torque measurement as the best trade-off value between negligible dynamic effect and torque signal stability. An waiting time interval of $20 \mathrm{~s}$ was used to let the system stabilize after each gas flow rate change, in each measurement position, and to avoid introducing in the bed long time range variations due to bed compaction.

Results in terms of experimental values of the torque as a function of the depth of the impeller and parametric in the aeration rate, represented as a fraction of the rate necessary for the system fluidization, are reported in Figure 6 for all the tested materials and for the two different impeller blade heights. Results tend to confirm qualitatively the trends of the torques observed by Bruni et al. as a function of the depth of the impeller and of the aeration rate. ${ }^{28}$ Higher spatial resolution profiles were obtained with the Anton Paar Powder Cell (Figure 6) with respect to previous studies. Inspection of the plots of the torque profiles highlights the existence of two regions for increasing powder bed depths: the first region closer to the bed surface is characterized by a linear increase of the torque with increasing depth. This trend is observed till a depth that is between 40 and $50 \mathrm{~mm}$ below the bed surface. A second region follows for deeper positions of the impeller where initially more curved and then flatter torque profiles are observed. Moreover, the effect of the air flow rate is to make the torque values lower for all the depths.

The prediction of the torque acting on the impeller in the Anton Paar Powder Cell can be achieved following the modeling approach by Tomasetta et al. ${ }^{16}$ Accordingly, the shearing surface around the impeller has been assumed to be shaped like the cylinder described by the impeller rotation, such as represented in Figure 7a. This cylinder has a height $h$ and diameter $d$, respectively, equal to the height of the impeller blade and to the double of the distance between the paddle tip and the shaft axis. It is assumed that the largest shearing action produced by the impeller is likely to be close to the impeller itself. Therefore, the estimated resistant torque, $T_{\bmod }$, will be the sum of the contributions of the stresses acting on the upper surface, $T_{\text {up }}$, the lower surface, $T_{\text {down }}$, and the lateral surface, $T_{\text {lateral }}$

$$
T_{\text {mod }}=T_{\text {up }}+T_{\text {down }}+T_{\text {lateral }}
$$

A cylindrical reference system will be used to express the torque as represented in Figure $7 \mathrm{~b}$. In this system, the axial coordinate, $z$, is vertical and has its origin at the bed surface and $r$ and $\theta$ are the radial and angular coordinates, respectively. Due to the small impeller height, the stress variations in the region around the impeller has been considered symmetrical with respect to the depth corresponding to the half of the impeller height. Thus, the stresses around the impeller had been equated to the stresses at a depth $z^{*}$ located at half of the height of the impeller blade in Figure 7a. Therefore, the torque contribution due to the shear at the upper and lower surfaces can be obtained from the integration of the shear stress on the horizontal and circular surface, $\tau_{z \theta}$

$$
T_{\text {up }}=T_{\text {down }}=\int_{o}^{\mathrm{d} / 2} \tau_{z \theta} 2 \pi r^{2} d r \approx \frac{\pi}{12} \tau_{z \theta}\left(z^{*}\right) d^{3}
$$

Furthermore, the torque contribution due to the shear on the lateral surface can be obtained from the integration of the shear stress on the vertical cylindrical surface, $\tau_{r \theta}$

$$
T_{\text {lateral }}=\frac{\pi}{2} d^{2} \int_{z *-\mathrm{h} / 2}^{z^{*}+\mathrm{h} / 2} \tau_{r \theta} d z \approx \tau_{r \theta}(z *) \frac{\pi}{2} d^{2} h
$$

Given the steady shearing, it might be argued that all the stresses at the impeller height and during its rotation are proportional to the vertical stress at $z^{*}, \sigma_{z}\left(z^{*}\right)$. In particular, an estimate of the horizontal stress, $\sigma_{r}$, can be obtained by making the hypothesis that

$$
\sigma_{r}=k_{i} \sigma_{z}
$$

Along the bed axis, both $\sigma_{r}$ and $\sigma_{z}$ are principal stresses. Therefore, if the material is internally mobilized, we can use the Mohr-Coulomb approximation to determine their ratio (Figure 8a) using the effective angle of internal friction $\delta$

$$
k_{i}=\frac{1-i \cdot \sin \delta}{1+i \cdot \sin \delta}
$$

where the coefficient $i$ is equal to 1 in case of active state of stress (the vertical stress is the major of the principal stresses), or it is equal to -1 in case of passive state of stress (the vertical stress is the minor of the principal stresses). Correspondingly, in the active state it is $k_{i}=k_{a}$ and in the passive state it is $k_{i}=k_{p}$. Furthermore, independently from the direction, we can assume a steady state shear stress on the shearing surface. The hypothesis of coaxiality between the stress and deformation (Figure 8b) on the shear plane (e.g., Ref. 29 or Ref. 30) assumes that the steady state shear stress is represented by the point of the Mohr circle in correspondence with the average stress. In fact, this position on the circle is the one providing a horizontal tangent on both the Mohr Circles of stress and strain, hypothesized to be coaxially oriented and, therefore, satisfying the condition of absence of powder dilation. Even if not often adopted in powder mechanics, the coaxiality condition provides an easy and, likely, correct order of magnitude to estimate the maximum possible shear stress in the steady state Mohr circle 

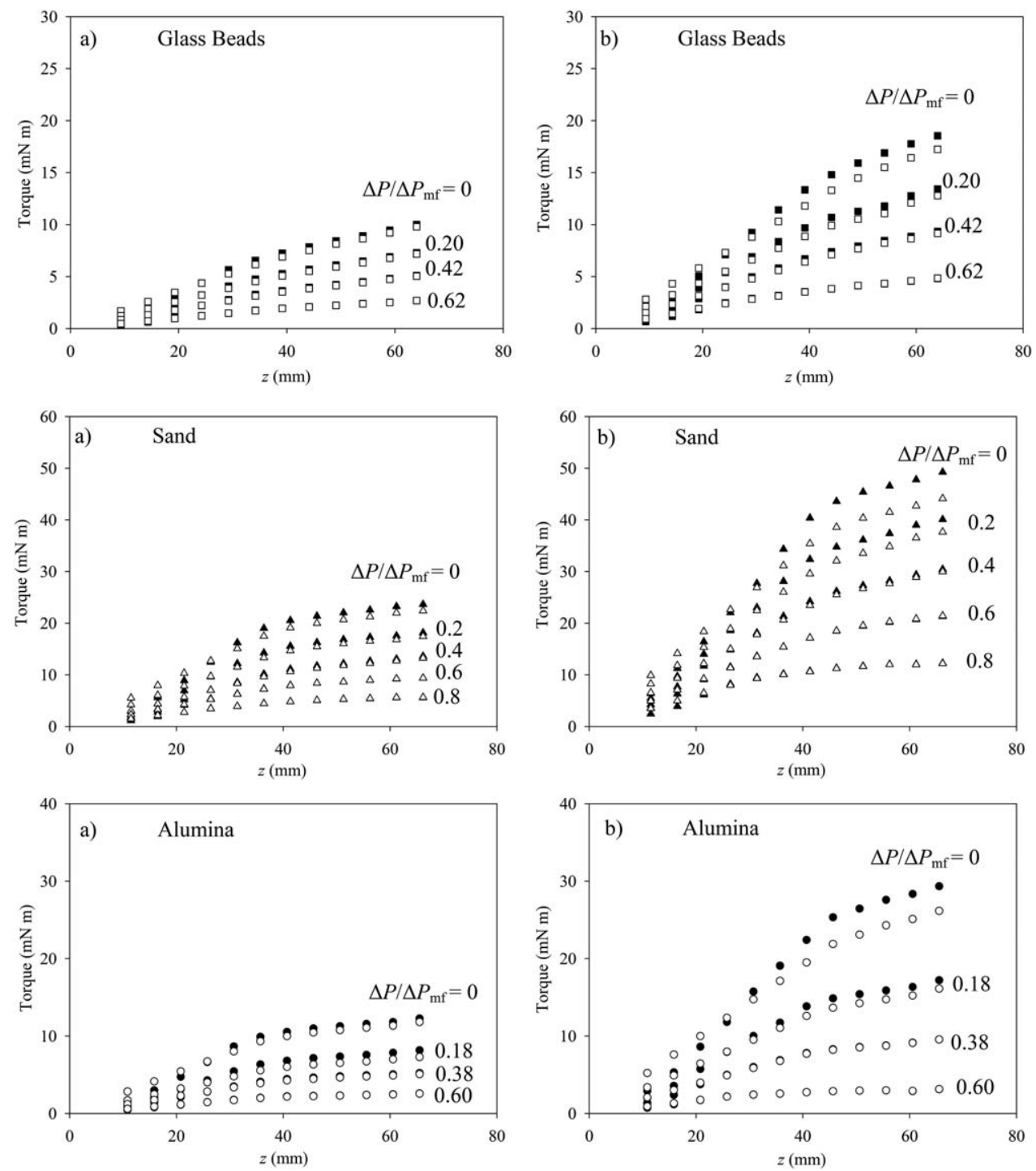

Figure 6. Torque measurements and model at different powder depths and volumetric flow rates for Glass beads ( $\square$ experimental results; $\square$ model results), sand $(\Lambda$, experimental results and $\triangle$, model results), and Alumina $(\boldsymbol{\bullet}$, experimental results and $\mathbf{0}$, model results).

(a) $5 \mathrm{~mm}$ height impeller and (b) $10 \mathrm{~mm}$ height impeller.

$$
\tau_{z \theta}=\sigma_{z} \sin \delta, \quad \tau_{r \theta}=\sigma_{r} \sin \delta
$$

Combining Eqs. 1-6 we have

$$
T_{\text {mod }}=\frac{\pi}{2} \sigma_{z}\left(z^{*}\right) d^{2} \sin \delta\left(\frac{d}{6}+h k_{i}\right)
$$

It appears clearly, from the derivation of Eq. 7 that the model has a number of simplifying hypotheses. Among these, one of the most significant is the shape and the extension of the shearing surface. For the sake of simplicity, we can introduce an empirical coefficient $\eta$ defined as the ratio between the experimental torque $T$ and the model torque $T_{\text {mod }}$ and attribute all the pitfalls between the model and the experiments to it

$$
T=\eta T_{\bmod }
$$

Most of the deviation of $\eta$ from 1 can be attributed to the fact that the real shearing surface is not as large as the shearing cylinder assumed in the model estimate. The parameter $\eta$ should be independent from the impeller depth, but it might be a function of the impeller extension and shape. It can be argued, in fact, that independently from what happens on the layers above the impeller, which determines the load at the impeller level, the state of stress at the impeller level is largely determined by the impeller motion. An indirect proof of this can be obtained by comparing the torque values measured by means of the full blade height impeller $\left(h^{\prime \prime}=10 \mathrm{~mm}\right)$ with the torque values measured by means of the half blade height impeller $\left(h^{\prime}=5 \mathrm{~mm}\right)$. The ratios of the two torques measured at all impeller depth are reported in Figure 9 for Glass Beads, Sand, and Alumina. Indicating with $T^{\prime \prime}$ and $T^{\prime}$ the two torque values and with $\eta^{\prime \prime}$ and $\eta^{\prime}$ the surface effectiveness extension in the two cases, from Eqs. 7 and 8 we have

$$
\frac{T^{\prime \prime}}{T^{\prime}}=\frac{\eta^{\prime \prime}}{\eta^{\prime}}\left(\frac{d}{6}+h^{\prime \prime} k_{i}\right) /\left(\frac{d}{6}+h^{\prime} k_{i}\right)
$$

Figure 10 reveals that experimental values of the torque ratio $T^{\prime \prime} / T^{\prime}$ are close to 2 . Beside the fact that $h^{\prime \prime}=2 h^{\prime}$, it can be assumed that for the same material $k_{i}$ is the same (the one for 

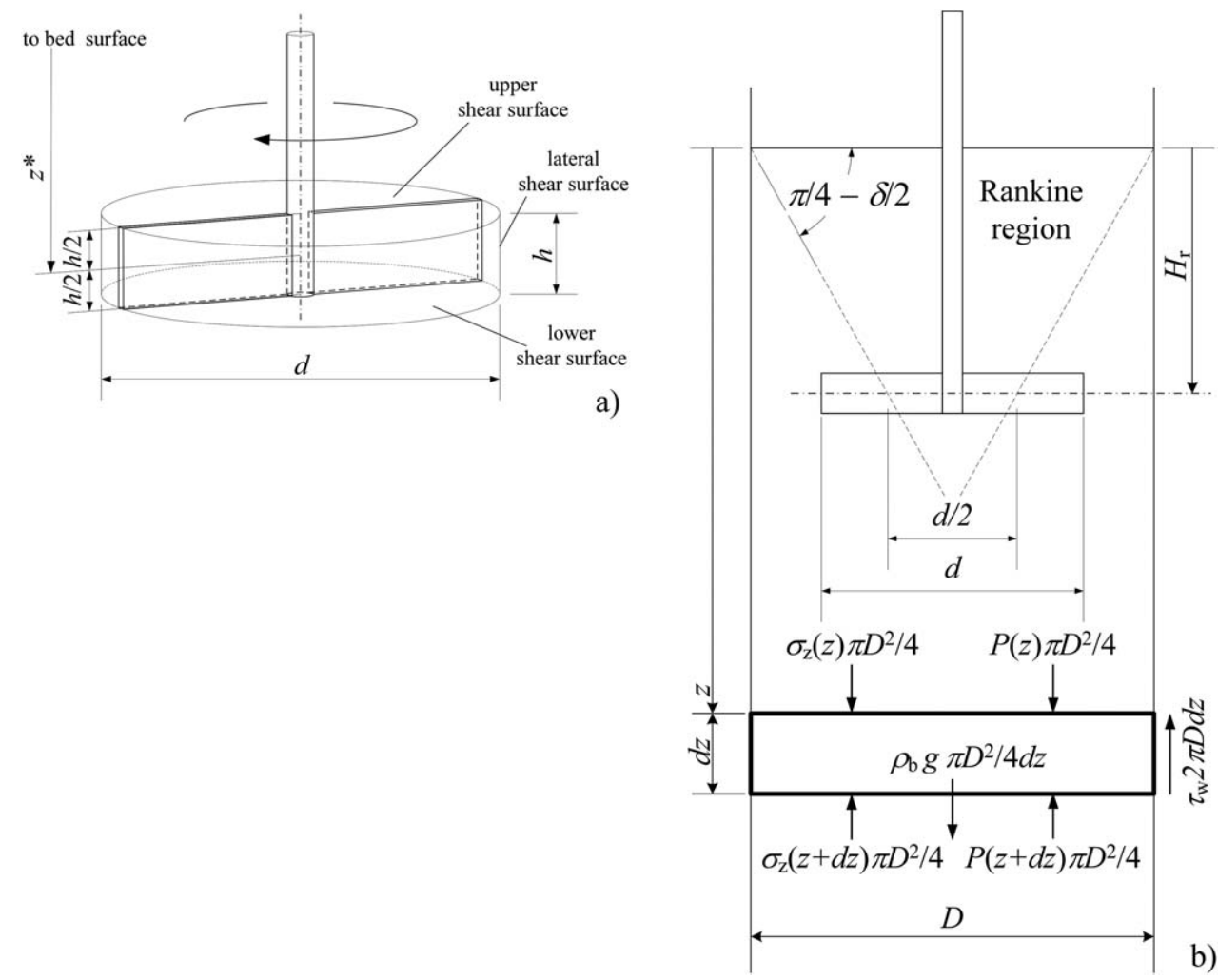

Figure 7. Model idealization:

(a) Sketch of the impeller ideal shearing surface. (b) reference system and force balance for the stress calculation above the impeller.

active or passive state) for the two different impellers tested and that the values of $\eta$ can change but not significantly. As a result, application of Eq. 9 suggests that $h k_{i} \gg d / 6$ or else $k_{i} \gg$ $d / 6 h$. Given the geometry of the system $(d=35 \mathrm{~mm}, h=10$ or $5 \mathrm{~mm}$ ), this implies necessarily values of $k_{i}>1$ that would suggest the occurrence of passive state of stress, at least at the level of the impeller. It is noteworthy that differently form the other materials the value the ratio $T^{\prime \prime} / T^{\prime}$ for alumina changes with aeration. This change can only be justified by a change of the ratio $\eta^{\prime \prime} / \eta^{\prime}$. A possible, yet speculative, explanation might be found in the fact that alumina is the finest material tested, the closer to an aeratable material among the tested ones. Therefore, it may happen that the concurrent action of internal shear and aeration can bring to different powder internal structure with aeration that can justify the change in the ratio $\eta^{\prime \prime} / \eta^{\prime}$.

The question that arises concerns the possibility to estimate $\eta$. A help might come from the data obtained with the impeller at the higher positions where an apparent linear dependency of the torque with the height is found. In the traditional Janssen approach, ${ }^{27}$ the vertical stress is affected by the gravitational body force acting on the powder, as well as by the wall tangential stresses, $\tau_{\mathrm{W}}$. The force balance acting on horizontal
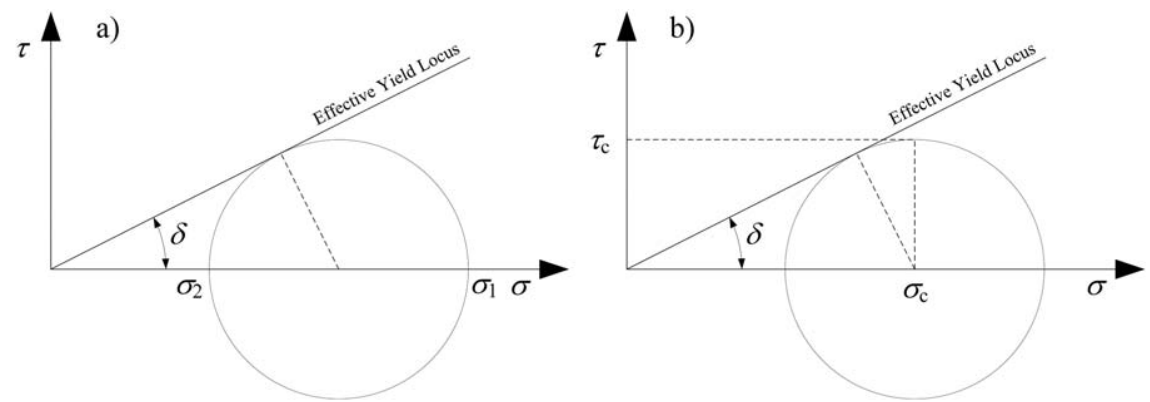

Figure 8. Graphical constructions: (a) at the base of Eq. 5 and (b) of Eq. 6. Pane

(a) reports a simplified representation of the state of stress within the bed above the impeller in the hypothesis of internal "mobilization." In the active state of stress $(i=1) \sigma_{1} \equiv \sigma_{z}$ and $\sigma_{2} \equiv \sigma_{r}$. In the passive state of stress $(i=-1) \sigma_{1} \equiv \sigma_{\mathrm{r}}$ and $\sigma_{2} \equiv \sigma_{z}$. Pane (b) reports a simplified representation of what is happening on the shearing surfaces. On the upper and lower shear surfaces of the cylinder representing the impeller shear envelope in Figure 6 we have $\sigma_{c} \equiv \sigma_{z}$ and $\tau_{c} \equiv \tau_{z \theta}$. On the lateral shear surfaces of the cylinder representing the impeller shear envelope in Figure 6 we have $\sigma_{c} \equiv \sigma_{r}$ and $\tau_{c} \equiv \tau_{r \theta}$. 

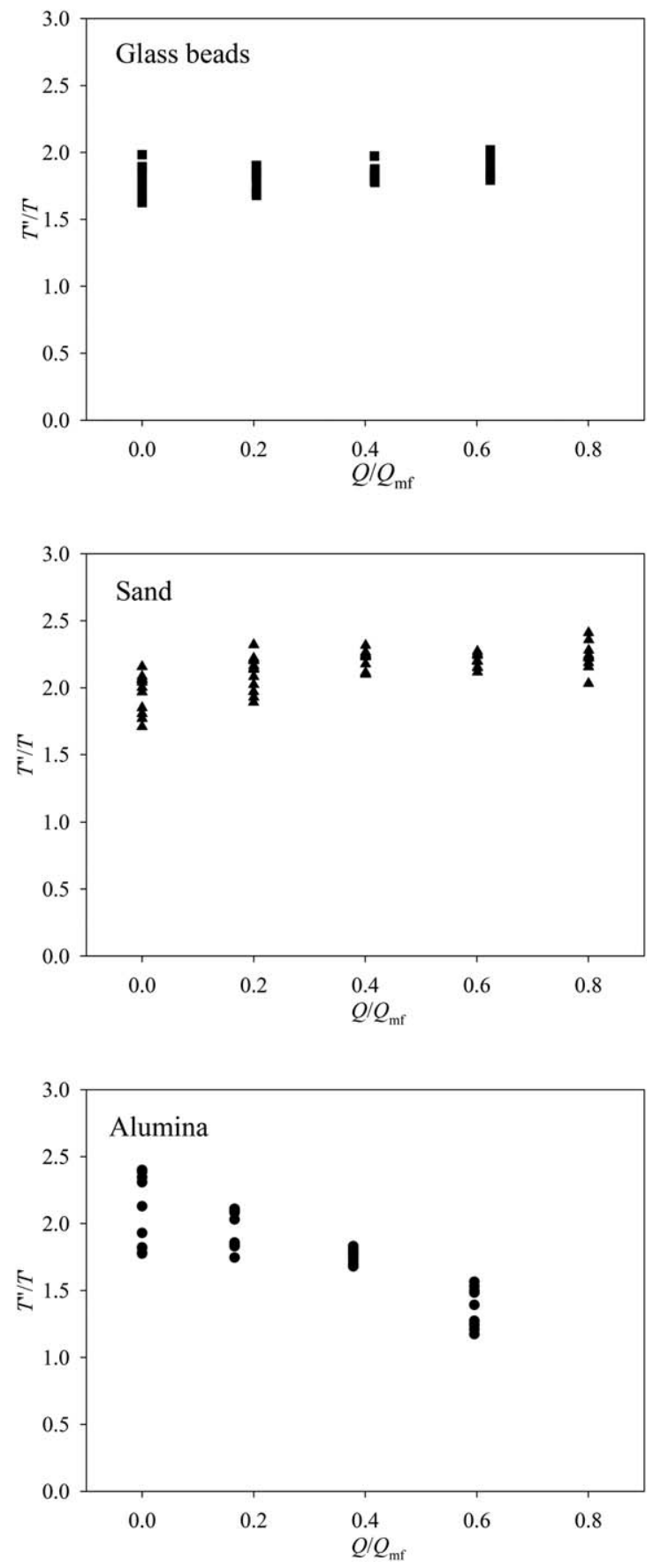

Figure 9. Ratio of torque measurements with the $10 \mathrm{~mm}$ impeller heights $\left(T^{\prime \prime}\right)$ to the torque measurements with the $5 \mathrm{~mm}$ impeller heights $\left(T^{\prime}\right)$ at different aeration rate for Glass beads, Sand, and Alumina.

elemental slices Figure 7b, on which the vertical stresses are assumed to be uniformly distributed, provides the following expression

$$
\frac{d \sigma_{z}}{d z}+\frac{4 \tau_{\mathrm{W}}}{D}=\rho_{\mathrm{b}} g-\frac{d P}{d z}
$$

where $D$ is the container inside diameter, $\rho_{\mathrm{b}}$ is the powder bulk density, $g$ is the acceleration due to gravity, $P$ is the local gas pressure, in case of vertical and uniform gas flow. As it appears from inspection of Eq. 10, a linear increase of the stress is only possible in case the wall effects are negligible. The stress model represented by Eq. 10, however, might be noncompletely adequate especially at the upper powder layers. In fact, the method of characteristics can be applied to estimate the stresses in a container. ${ }^{30}$ According to this method, a conical core of the upper layers of the powder is apparently unaffected by the presence of the wall. This conical region is called Rankine region and is included by the inward characteristics surfaces starting, unaffected by the wall, at the junction between the free material surface and the walls, as shown in Figure $7 \mathrm{~b}$. The inclination of the characteristics at the free surface is at an angle with the horizontal plane of $\pi / 4+\delta / 2$. Inside the Rankine region, where the stresses are unaffected by the wall presence, it is reasonable to assume a sort of hydrostatic state of stress, in which the vertical stresses are proportional to the depth. According to this interpretation, the impeller, would provide a torque that is proportional to its depth below the surface down to a depth that is the one at which the impeller blade significantly crosses the external surface of the conical Rankine region, say for half of its length. This would produce impeller depth expressed by the following expression

$$
H_{r}=\left(D-\frac{d}{2}\right) \tan \left(\frac{\pi}{4}+\frac{\delta}{2}\right)
$$

Values of these depths are provided in Table 1 and compare rather satisfactorily with the depth at which a transition is observed in the torque profiles between a linear profile and a curved profile in Figure 6. It should be noted that, similarly to the experiments, this transition is not significantly dependent on the aeration rate through the powder sample. It is also noteworthy that the slope of the characteristics which provides a good agreement between this model interpretation and the experiments requires an active state of stress in the bed above the impeller, while, at the impeller level, due to the impeller action we can assume a passive state of stress.

As a result, in the Rankine zone, where $z<H_{r}$ we have a hydrostatic dependency of the state of stress

$$
\sigma_{z}(z)=\left(\rho_{\mathrm{b}} g-\frac{\Delta P_{\mathrm{b}}}{H_{\mathrm{b}}}\right) \cdot z
$$

Therefore Eq. 12 allows to estimate $T_{\text {mod }}$ with Eq. 7, in which $k_{i}=k_{a}$, and Eq. 8 can be used to calculate $\eta$. Figure 10 reports

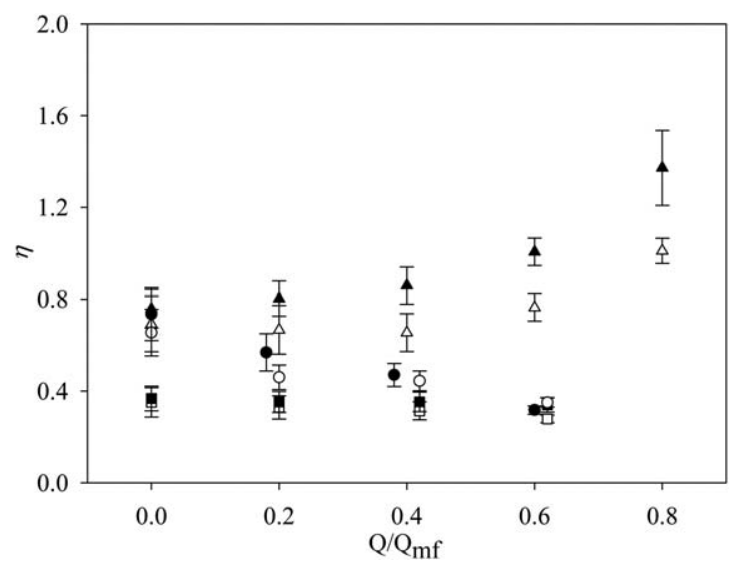

Figure 10. Factor $\eta$ at different aeration rates of Glass beads $\square, 10 \mathrm{~mm}$ impeller; $\square, 5 \mathrm{~mm}$ impeller), Sand $(\Delta, 10 \mathrm{~mm}$ impeller; $\triangle, 5 \mathrm{~mm}$ impeller), and Alumina $(0,10 \mathrm{~mm}$ impeller; $\bigcirc, 5 \mathrm{~mm}$ impeller). 

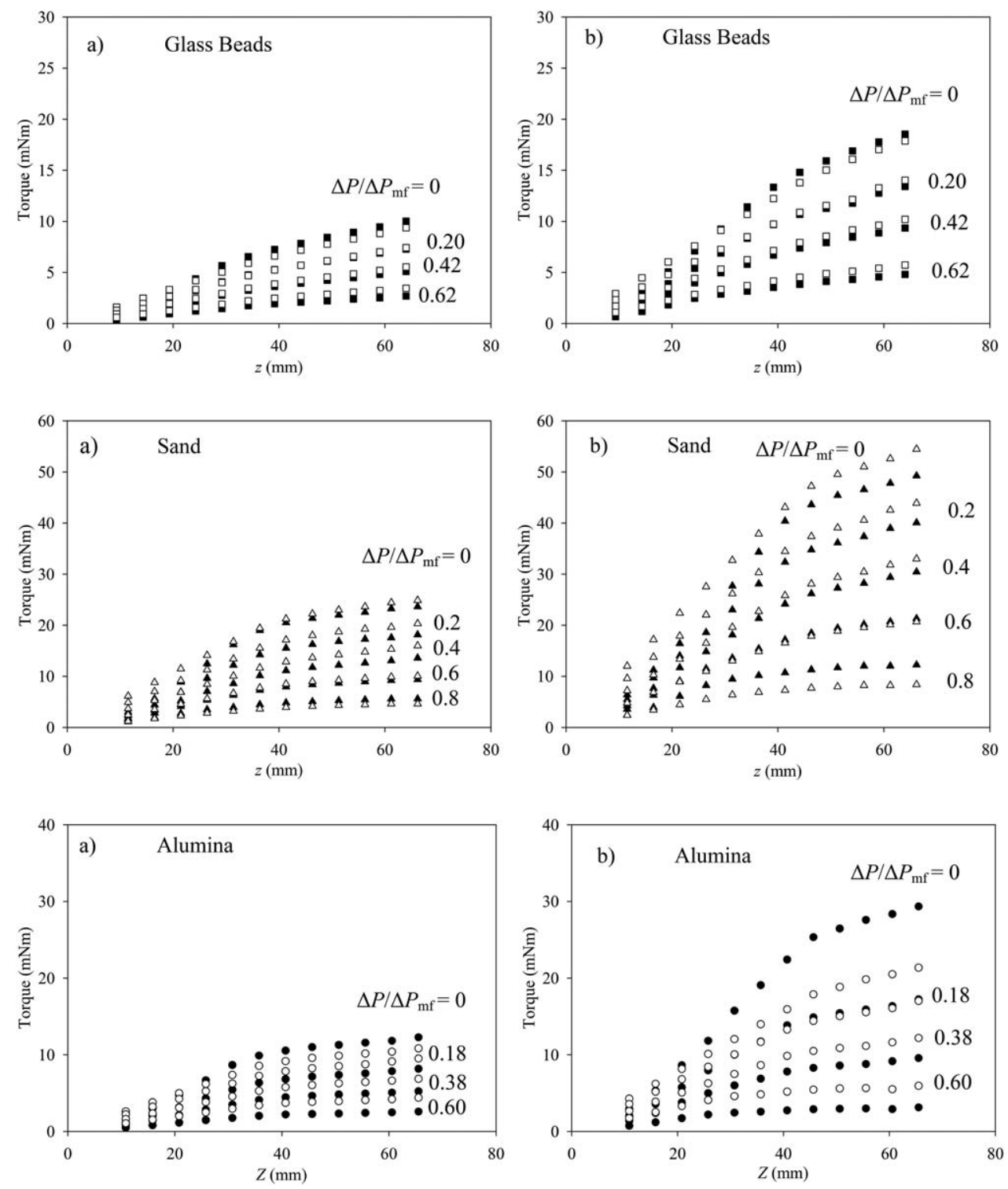

Figure 11. Torque measurements and model at different powder depths and volumetric flow rates with fixed $\eta$.

(a) $5 \mathrm{~mm}$ height impeller and (b) $10 \mathrm{~mm}$ height impeller. Glass beads ( $\square$ experimental results; $\square$ model results) $(\eta=0.42)$, Sand $(\Lambda$, experimental results; $\triangle$, model results) $(\eta=1)$ and Alumina $(\boldsymbol{\bullet}$, experimental results; $\boldsymbol{O}$, model results) $(\eta=0.6)$.

the values of $\eta$ for the different materials and as a function of the aeration rate used. Results indicate a rather strong dependence of $\eta$ on the specific material tested and a much slighter dependence of this parameter on the height of the impeller blade as well as of the aeration rate. In particular, $\eta$ values of about $0.7-0.8$ are obtained for sand and alumina under nonaerated conditions $\left(Q / Q_{\mathrm{mf}}=0\right)$, indicating a good agreement between model and experiments. A gradual increase of $\eta$ up to values larger than 1 is observed for sand with increasing air flow rate beyond $Q / Q_{\mathrm{mf}}=0.2$. Instead, a progressive decrease of $\eta$ up to values lower than 0.4 is observed for alumina with increasing air flow rate. Differently, $\eta$ values are generally about 0.4 for glass beads for all aeration conditions indicating a general overestimate of the torque by the model.

As above mentioned most of the values of $\eta$ are smaller than 1 suggesting the interpretation that $\eta$ might account for the reduced extension of the effective shear plane surface with respect to the ideal cylinder defined by the revolution of the impeller blade. However, values of $\eta$ larger than 1 may indicate that the effective stress acting on the shear surface can be larger than the one predicted by our simple model. A possible reason for this higher stress values might come from particle jamming phenomena between the impeller tip and the wall. Jamming effects may reasonably be more significant for coarser materials with a larger angle of internal friction such as sand within our materials. We have looked for a possible support to this explanation in the literature, but such phenomena seems to be not very well documented, even in the rather well developed literature devoted to the mechanics of powder in shear granulators. Novosad ${ }^{31}$ showed that the shear plane can extend further the tip of the blade toward the external wall, but does not quantify the stress effect. In their experiments on the effect of the bottom wall to the kinematics and to the mechanics of the powder shear induced by the movement of a blade, Bagster and Bridgwater ${ }^{32,33}$ did find some limited effect. In that case, however, the geometry defined by the 
impeller and the wall was somewhat different than the one considered in this article. As a further remark, the larger value of $\eta^{\prime \prime}$ with respect to $\eta^{\prime}$ can be consistent with the interpretation of the presence of particle jamming between the blade tip and the wall. In fact, considering that particle close to the blade tip corner can more easily escape the jamming region between the blade tip and the wall by shifting upward or downward, it can be easily understood that the same escape mechanism can be more relevant for the lower height blade, producing a less significant increment of $\eta$ as, in fact, is observed. Also, the observed increase of $\eta$ with air flow rate for sand in Figure 10 is consistent with the higher relative importance of the jamming phenomena in aerated conditions when the shear stresses on the shear planes, other than those in the jamming regions are reduced.

It is interesting and still consistent with the above framework of interpretation that the values of $\eta$ for the two different impeller blade height are almost similar for glass beads in Figure 10. In fact, for this material the registered values of $\eta$ seem to indicate that jamming would not occur as significantly as for sand.

Considering the above observation for alumina on the change of the ratio $T^{\prime \prime} / T^{\prime}$ with air flow rate, it appears from Figure 10 that this material is the only one in which the differences between $\eta^{\prime}$ and $\eta^{\prime \prime}$ change with the air flow rate. As above mentioned, the change of the relative value of $\eta^{\prime}$ and $\eta^{\prime \prime}$ might depend on the higher sensitivity of this material to change the internal structure due to the air effect. It is significant the fact that instead for glass beads $\eta^{\prime}$ and $\eta^{\prime \prime}$ are almost unaffected by air flow rate and by the impeller size.

To obtain a profile of the vertical stress Eq. 10 can be piecewise integrated beyond the Rankine zone for $z>H_{r}$ as follows

$$
\sigma_{z}(z+\Delta z)=\sigma_{z}(z)+\left(\rho_{\mathrm{b}} g-\frac{\Delta P_{\mathrm{b}}}{H_{\mathrm{b}}}-\frac{4 \sigma_{\mathrm{z}}(z) k_{a} \tan \varphi_{\mathrm{w}}}{D}\right) \Delta z
$$

In Eq. 13, the local gas pressure gradient has been replaced by the average gas pressure gradient in the bed obtained by dividing the bed gas pressure drop, $\Delta P_{\mathrm{b}}$, by the bed height, $H_{\mathrm{b}}$. Furthermore, the wall friction has been related to the radial stress using the full mobilization hypothesis of the material at the wall and considering a constant wall friction value $\phi_{\mathrm{w}}$ for each material

$$
\tau_{\mathrm{w}}=\sigma_{r} \tan \varphi_{\mathrm{w}}
$$

Equations 12 and 13 allow to estimate $T_{\bmod }$ with Eq. 7, in which $k_{i}=k_{p}$, the effective angle of internal friction $\delta$ was taken as the value measured for the material and reported in Table 1 and the wall friction angle is assumed as a single fitting parameter $\left(\phi_{\mathrm{w}}=\phi_{\mathrm{we}}\right)$, for all the experiments performed with the same material. In turn, $T_{\text {mod }}$ can be corrected with Eq. 8 using the values of $\eta$ found in the Rankine region for each material and each aeration rate. The results of the application of this model are compared with the experimental results in Figure 6. Table 1 reports the $\phi_{\text {we }}$ values and, for the sake of comparison, the wall friction values $\phi_{\mathrm{wm}}$ measured with the Brookfield PFT on wall samples made of plane glass. The agreement between model and experiments in Figure 6 is fairly good. Conversely, the comparison in Table 1 between $\phi_{\mathrm{we}}$ and $\phi_{\mathrm{wm}}$ indicates that the wall friction required by the model to predict the experimental torque profile is always lower than the directly measured material wall friction. This latter finding may suggest that the material at the wall is not necessarily fully mobilized. Overall the physical interpretation provided for these experiments seems to be supported by the provided results.

To assess the sensitivity of calculated results to the specific values of $\eta$, Figure 11 reports the model results for the torque in which for each material a single value of $\eta$ was used for all the aeration rates. It can be seen that, the less satisfactory results are obtained with sand and the higher blade and with alumina for both the blade heights. The result for sand is consistent with the hypothesis that a single value of $\eta$ cannot account for the change of weight of the effect of particle jamming between the blade tip and the walls for this material. Also for alumina the unsatisfactory result is expected given the aforementioned interpretation of the possible change of the internal structure of the bed with air and shear which can produce significant changes in the powder internal structure.

\section{Conclusions}

The Anton Paar Powder cell is able to precisely measure the torque values necessary to rotate an impeller in aerated beds in the full range of aeration between no aeration and the minimum for fluidization. Measured torque values depend on the material tested, on the air flow rate applied, on the impeller depth and on the height of the impeller blade. A minor effect of the impeller rotation speed is verified below the rotational speed of $0.6 \mathrm{rpm}$. The impeller depth seems to affect linearly the torque for low impeller depth. Instead, the torque increases less than linearly at high depth values.

A model was developed for the interpretation of results based on the idea that the material is shearing on the impeller surface described by the impeller rotation. The linear dependence of the torque on the impeller depth might be related to the presence of a Rankine conical region, on the bed top, in which the stress state is not affected by the presence of the container walls. The effectiveness of the model prediction of the torque was estimated for the impeller within the Rankine region. Constant values of the effectiveness used together with the Janssen one-dimensional force balance in the container allow to correctly estimate torque changes below the Rankine region. These results suggest that the torque measured with Anton Paar Powder cell can be directly related to the powder properties at low consolidation stresses. These stresses can be as low as those determined by the weight of powder layers of few millimeters or an by the effective weight of deeper powder beds that accounts for the lifting effect of aeration.

Further research should be addressed to use the developed model predictively. In particular, rheometer measures should be directly correlated to some powder flow properties, such as the effective angle of internal friction, by means of the model application. One of the objective of the research should be the possible sources of unexpected changes of the torque possibly due to particle jamming phenomena between the blade tip and the walls. Experiments involving changes in the gap between the blade tip and the walls should shed some light on this point. Finally, a more complex open question regards how would be to correctly account for the possible change of the internal structure of aeratable powders such as alumina with air. When this happen it is not possible to find a unique value of the torque calibration factor at all aeration rates but, as proposed, the calibration factor should be evaluated at each of the aeration rates tested. 


\section{Notation}

$A=$ Anton Paar Powder Cell cross-section area, $\mathrm{m}^{2}$

$h=$ impeller height and height of the shearing cylinder around the impeller, $\mathrm{m}$

$d=$ Impeller diameter, $\mathrm{m}$

$D=$ container inside diameter, $\mathrm{m}$

$d_{\mathrm{p}}=$ particle diameter, $\mathrm{m}$

$d_{\mathrm{p} 10}=$ particle diameter corresponding to the 10th percentile of the cumulative particle size distribution in weight, $\mathrm{m}$

$d_{\mathrm{p} 3,2}=$ Sauter mean diameter, $\mathrm{m}$

$d_{\mathrm{p} 50}=$ particle diameter corresponding to the median of the cumulative particle size distribution in weight, $\mathrm{m}$

$d_{\mathrm{p} 90}=$ particle diameter corresponding to the $10^{\text {th }}$ percentile of the cumulative particle size distribution in weight, $\mathrm{m}$

$g=$ acceleration due to gravity, $\mathrm{m} \mathrm{s}^{-2}$

$H_{\mathrm{b}}=$ bed height, $\mathrm{m}$

$k_{a}=$ ratio between the radial and the axial normal stress at active state, dimensionless

$k_{p}=$ ratio between the radial and the axial normal stress at passive state, dimensionless

$M=$ mass of powder in the bed, $\mathrm{kg}$

$p=$ gas pressure, $\mathrm{Pa}$

$r=$ radial coordinate in the Anton Paar Powder Cell, $\mathrm{m}$

$T$ = experimental torque, $\mathrm{N} \mathrm{m}$

$T_{\text {down }}=$ torque acting on the lower surface of the shearing cylinder, $\mathrm{N} \mathrm{m}$

$T_{\text {lateral }}=$ torque acting on the lateral surface of the shearing cylinder, $\mathrm{N} \mathrm{m}$

$T_{\text {mod }}=$ the estimated torque

$T_{\text {up }}=$ torque acting on the upper surface of the shearing cylinder, $\mathrm{N}$ $\mathrm{m}$

$U_{\mathrm{mf}}=$ minimum air velocity for attaining constant pressure drop

$z=$ the axial coordinate, -

$z^{*}=$ impeller depth, $\mathrm{m}$

$H_{r}=$ Efffective depth of the rankine region, $\mathrm{m}$

\section{Greek letters}

$\phi_{\mathrm{w}}=$ angle of wall friction, deg.

$\phi_{\mathrm{we}}=$ measured angle of wall friction, deg.

$\phi_{\mathrm{wm}}=$ model estimates of the angle of wall friction, deg.

$\theta=$ angular coordinates, -

$\tau_{z \theta}=$ shear stress on the horizontal and circular surface, $\mathrm{N} \mathrm{m}$

$\tau_{r \theta}=$ shear stress on the vertical cylindrical surface, $\mathrm{N} \mathrm{m}$

$\tau_{\mathrm{W}}=$ wall tangential stresses

$\sigma_{r}=$ horizontal stress, $\mathrm{N} \mathrm{m}$

$\sigma_{z}=$ vertical stress, $\mathrm{N}$ m

$\delta=$ effective angle of internal friction, deg.

$\eta=$ torque ratio between experiments and theory of cylindrical shearing surface, -

$\rho_{\mathrm{b}}=$ powder bulk density, $\mathrm{kg} \mathrm{m}^{-3}$

$\Delta P_{\mathrm{b}}=$ bed gas pressure drop, $\mathrm{Pa}$

\section{Literature Cited}

1. Léonard G, Abatzoglou N. Stress distribution in lubricated vs unlubricated pharmaceutical powder columns and their container walls during translational and torsional shear testing. Powder Technol. 2010;203(3):534-547.

2. Harnby N. An engineering view of pharmaceutical powder mixing. Pharm Sci Technol Today. 2000;3(9):303-309.

3. Suri A, Horio M. A novel cartridge type powder feeder. Powder Technol. 2009;189(3):497-507.

4. Daniher DI, Zhu J. Dry powder platform for pulmonary drug delivery. Particuology 2008;6(4):225-238. doi:10.1016/j.partic.2008.04.004.

5. Fitzpatrick J, Barringer S, Iqbal T. Flow property measurement of food powders and sensitivity of Jenike's hopper design methodology to the measured values. J Food Eng. 2004;61(3):399-405.

6. Hassanpour A, Ghadiri M. Characterisation of flowability of loosely compacted cohesive powders by indentation. Part Part Syst Charact. 2007;24(2):117-123.

7. Vinter S, Pedersen T, Allesø M, Garnaes J, Rantanen J. Evaluation of ring shear testing as a characterization method for powder flow in small-scale powder processing equipment. Int J Pharm. 2014; 475(1-2):315-323.

8. Salehi H, Barletta D, Poletto M. A comparison between powder flow property testers. Particuology. 2017;32:10-20.

9. Schulze D. Powders and Bulk Solids. Berlin, Heidelberg: SpringerVerlag, 2008:156-160. doi:10.1007/978-3-540-73768-1.
10. Schwedes JJ. Review on testers for measuring flow properties of bulk solids. Granul Matter. 2003;5(1):1-43.

11. Schulze D, Wittmaier A. Flow properties of highly dispersed powders at very small consolidation stresses. Chem Eng Technol. 2003; 26(2):133-137.

12. Klein J, Höhne D, Husemann K. The influence of air permeation on the flow properties of bulk solids. Chem Eng Technol. 2003;26(2): $139-146$.

13. Johanson K, Barletta D. The influence of air counter-flow through powder materials as a means of reducing cohesive flow problems. Part Part Syst Charact. 2004;21(4):316-325.

14. Barletta D, Donsì G, Ferrari G, Poletto M. A rotational tester for the characterization of aerated shear flow of powders. Part Part Syst Charact. 2007;24(4-5):259-270.

15. Vasilenko A, Koynov S, Glasser BJ, Muzzio FJ. Role of consolidation state in the measurement of bulk density and cohesion. Powder Technol. 2013;239:366-373.

16. Tomasetta I, Barletta D, Lettieri P, Poletto M. The measurement of powder flow properties with a mechanically stirred aerated bed. Chem Eng Sci. 2012;69(1):373-381.

17. Castellanos A, Valverde J, Quintanilla M. The Sevilla powder tester: a tool for characterizing the physical properties of fine cohesive powders at very small consolidations. KONA Powder and Particle J. 2004;22(22):66-81. Available at: http://www.kona.or.jp/search/22_ 066.pdf.

18. Valverde JM, Castellanos A, Ramos A, Pérez AT, Morgan MA, Keith Watson P. An automated apparatus for measuring the tensile strength and compressibility of fine cohesive powders. Rev Sci Instrum. 2000;71(7):2791.

19. Castellanos A, Quintanilla M, Valverde JM, Soria-Hoyo C. Novel instrument to characterize dry granular materials at low consolidations. Rev Sci Instrum. 2007;78(7):73901.

20. Zafar U, Hare C, Calvert G, Ghadiri M, Girimonte R, Formisani B, Quintanilla QAS, Valverde JM. Comparison of cohesive powder flowability measured by Schulze Shear Cell, Raining Bed Method, Sevilla Powder Tester and new Ball Indentation Method. Powder Technol. 2015;286:807-816.

21. Bruni G, Colafigli A, Lettieri P, Elson T. Torque measurements in aerated powders using a mechanically stirred fluidized bed rheometer (msFBR). Chem Eng Res Des. 2005;83(11):1311-1318.

22. Freeman R. Measuring the flow properties of consolidated, conditioned and aerated powders — a comparative study using a powder rheometer and a rotational shear cell. Powder Technol. 2007;174(12):25-33.

23. Leturia M, Benali M, Lagarde S, Ronga I, Saleh K. Characterization of flow properties of cohesive powders: a comparative study of traditional and new testing methods. Powder Technol. 2014;253:406-423.

24. Hare C, Zafar U, Ghadiri M, Freeman T, Clayton J, Murtagh MJ. Analysis of the dynamics of the FT4 powder rheometer. Powder Technol. 2015;285:123-127.

25. Nan W, Ghadiri M, Wang Y. Analysis of powder rheometry of FT4: effect of air flow. Chem Eng Sci. 2017;162:141-151.

26. Bruni G, Lettieri P, Newton D, Barletta D. An investigation of the effect of the interparticle forces on the fluidization behaviour of fine powders linked with rheological studies. Chem Eng Sci. 2007;62(12):387-396.

27. Sperl M. Experiments on corn pressure in silo cells - translation and comment of Janssen's paper from 1895. Granul Matter. 2006;8(2): $59-65$.

28. Bruni G, Barletta D, Poletto M, Lettieri P. A rheological model for the flowability of aerated fine powders. Chem Eng Sci. 2007;62(12):397-407.

29. Schulze D. Powders and Bulk Solids. Berlin, Heidelberg: Springer Verlag, 2008:149.

30. Nedderman RM. Statics and Kinematics of Granular Materials. Cambridge University Press, 1992:166.

31. Novosad J. Studies on granular materials. III. Stress distribution in a granular material mixed by a mechanical impeller. Collect Czech Chem Commun. 1964;29(12):2702-2709.

32. Bagster DF, Bridgwater J. The flow of granular material over a moving blade. Powder Technol. 1969;3(1):323-338.

33. Bagster DF, Bridgwater J. The measurement of the force needed to move blades through a bed of cohesionless granules. Powder Technol. 1967;1(4):189-198. 